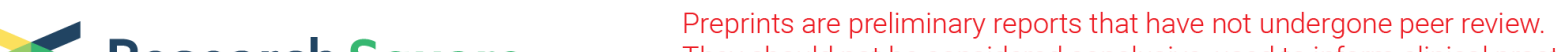 Research Square They should not be considered conclusive, used to inform clinical practice, or referenced by the media as validated information.
}

\section{BVD protects against UVB-induced HaCaT keratinocytes photodamage through reactivating Nrf2 antioxidative stress signaling}

\section{Ma Liangjuan}

Second Affiliated Hospital of Harbin Medical University

Gong Jie

Second Affiliated Hospital of Harbin Medical University

\section{Liu Yingdi}

Chifeng Municipal Hoapital

Ren Jing

Caxian People's Hospital

\section{Lin Fang}

Cangzhou Central Hospital

\section{Zhang Xiaohui}

Weifang Traditional Chinese Hospital

\section{You Yan}

Harbin Medical University Fourth Hospital

\section{Bing xue Bai ( $\nabla$ bxuebdd@163.com )}

Second Affiliated Hospital of Harbin Medical University https://orcid.org/0000-0003-1044-488X

\section{Li Yuzhen}

Second Affiliated Hospital of Harbin Medical University

\section{Research article}

Keywords: Biliverdin, UVB irradiation, Photo-damage, Nrf2, oxidativestress, HaCaT

Posted Date: February 25th, 2020

DOl: https://doi.org/10.21203/rs.2.24395/v1

License: (c) (1) This work is licensed under a Creative Commons Attribution 4.0 International License. Read Full License 


\section{Abstract}

Background : To investigate the protective role and mechanism of exogenous biliverdin (BVD) on ultraviolet B (UVB) irradiated human keratinocytes (HaCaT cells).

Methods : Cultured HaCaT cells were divided into control group, UVB group, and BVD + UVB group. Morphological changes in the HaCaT cells were observed, the cell viability of each group was detected, the mean fluorescence intensity (reactive oxygen species, ROS) of the cells was detected, the superoxide dismutase (SOD) and malondialdehyde (MDA) levels of the cells were detected, and the protein expression levels of nuclear factor erythroid 2-related factor 2 (Nrf2), matrix metalloproteinase-1 (MMP-1), and matrix metalloproteinase-3 (MMP-3) were determined. Moreover, Nrf2 gene transfection was performed in HaCaT cells.

Results : UVB irradiation induced apoptosis of HaCaT cells, decreased Nrf2 protein expression and increased MMP-1 and MMP-3 protein expression, which increased ROS and MDA levels and decreased SOD levels. Nrf2 gene enhancement increased UVB-irradiated HaCaT cell viability.

Conclusion : Exogenous BVD plays a protective role in UVB-induced HaCaT cell damage, and this effect may be related to the Nrf2 antioxidant signaling pathway.

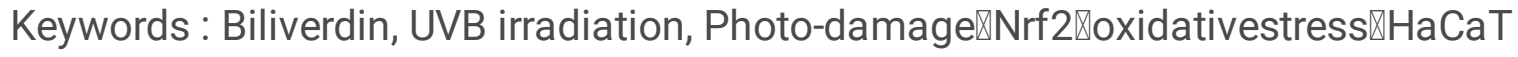

\section{Background}

Ultraviolet (UV) irradiation causes photoaging of the skin [1]. UV radiation is divided into UVA (320$400 \mathrm{~nm})$, UVB (280-320 nm), and UVC (100-280 nm), wherein UVC is absorbed by the ozone layer [2], while UVA and UVB are important factors that cause photoaging of the skin. UVA, with the longest wavelength, is the tanning segment; although it does not cause acute inflammation of the skin, it has strong penetrating power that can easily damage the dermis of the skin and destroy fibrous tissue, and long-term accumulation can age the skin, resulting in wrinkles. UVB can destroy the moisturizing capability of the skin, making the skin rough and wrinkled, and long-term irradiation can thicken the skin keratin and cause erythema, inflammation, skin aging and, in severe cases, skin cancer. UV rays can induce photoaging of the skin by inducing the generation of reactive oxygen species (ROS) and malondialdehyde (MDA) through lipid peroxidation, inhibiting the activity of superoxide dismutase (SOD), inducing the expression of metalloproteinases, and inducing the activation of protein kinase signaling pathways $[3,4]$. In the skin, UV rays can destroy the normal regulatory function of the mitochondria, which induces a large increase in ROS. A large amount of ROS can degrade polyunsaturated fatty acids in cell membranes, causing lipid peroxidation [5]; lipid peroxidation is generally considered to be a marker of induced cellular oxidative damage. MDA is the final decomposition product of lipid peroxidation triggered by free radicals. MDA is the most widely recognized marker of lipid peroxidation and oxidative stress [6]. UV rays can directly or indirectly generate effects on the body's oxidation and antioxidation balance. SOD is a chief superoxide free radical clearing factor that naturally exists in the body, and its 
high or low level in organisms is related to cell senescence and death [7]. UV irradiation can trigger a series of conduction changes in intracellular signal pathways and activate the expression of downstream matrix metalloproteinases (MMPs). MMPs degrade almost all extracellular matrix components, which causes degradation of the normal collagen elements and elastic fibers and ultimately leads to photoaging $[8,9]$.

Nuclear factor erythroid 2-related factor 2 (Nrf2) is a transcription factor that has a basic leucine zipper structure; it is the main regulatory factor of antioxidant responses [10]. As a redox-sensitive transcription factor, Nrf2 mediates a series of cytoprotective factors, including antioxidant enzymes such as heme oxygenase-1 (HO-1) and SOD [11]. It has been reported that Nrf2 deletion or activation disorder increases the sensitivity of cells to UV irradiation and that the Nrf2/ARE pathway plays a protective role in cell photodamage [12-14]. Studies in recent years have shown that Nrf2 protein expression is reduced in $\mathrm{HaCaT}$ cells after UVB irradiation and that the application of an Nrf2 activator can effectively activate the Nrf2 antioxidative stress pathway, which participates in reducing oxidative stress [15]. Therefore, protecting against UV-induced oxidative stress by activating Nrf2 provides a new line of thinking in the prevention and treatment of photoaging.

Heme oxygenase-1 (HO-1) degrades heme into carbon monoxide, BVD and free iron. BVD is rapidly converted to bilirubin (BIL) by the enzyme biliverdinreductase. Indeed, accumulating evidence indicates that BVD/BIL has a wide spectrum of biological effects such as antioxidant, anti-inflammatory and cytoprotective effects[16]. At present, studies regarding BVD and its metabolite BIL are mainly concentrated in fields related to the protection of the nervous system, the cardiovascular system, and organ transplantation $[17,18]$. However, there are no other researchers who have reported on the action of BVD in skin photoaging and cell photodamage. It has been reported in studies that BVR in skin fibroblasts decreases with age [19]; therefore, it may play a protective role in skin photoaging through the related metabolites BVD/BIL $[20,21]$. In a previous animal experiment, we confirmed that exogenous BVD can delay the UV-induced chronic skin photoaging in nude mice [22]. In the current study, we studied the antioxidative and antiaging role of BVD in UVB-induced $\mathrm{HaCaT}$ cell damage, and explored the mechanism of BVD antagonization of skin photodamage by observing the effect of BVD on Nrf2. In addition, we observed the severity of cellular damage from UV rays through activating or silencing the Nrf2 gene to further prove that Nrf2 may be related to the antioxidative stress pathway.

\section{Results}

Morphological changes in HaCaT cells before and after UVB irradiation

Under observation with an inverted optical microscope, HaCaT cells presented a flat paving stone-like morphology, and cell growth was vigorous (Fig. 1a); 24 hours after irradiation with $30 \mathrm{~mJ} / \mathrm{cm}^{2} \mathrm{UVB}$, $\mathrm{HaCaT}$ cells became smaller and rounded, and the numbers were clearly reduced (Fig. 1b). 
The CCK-8 results show that the cell viability of experimental groups was $100 \%, 32.93 \%, 48.85 \%, 52.92 \%$, and $64.74 \%$ (Fig. 2), respectively. Compared with the control group, cell activity in the UVB group was clearly reduced, and the difference was statistically significant $(p<0.05)$; compared with the UVB group, the cell viability of BVD pretreatment group increased significantly. Moreover, as the BVD concentration increased, the cell viability also increased, presenting a dosage correlation, and the difference for each BVD experimental group was statistically significant $(p<0.05)$.

Effect of BVD on ROS fluorescence intensity,SOD and MDA of UVB-irradiated HaCaT cells

A flow cytometer was used to detect intracellular ROS levels (Fig. 3a-f). Approximately 10,000 cells were counted per gate to calculate the mean fluorescence intensity of a single cell (MEAN), and P2 was the region indicating positive ROS expression. Compared with the control group, the mean ROS fluorescence intensity of the cells in the UVB group clearly increased, indicating that more ROS was generated in the cells after UVB irradiation, which caused certain damage to the cells. The ROS level (MEAN) of the cells incubated with BVD prior to UVB irradiation was clearly lower than that of the UVB group, indicating a dose-dependent correlation with BVD concentration. The results in Fig. $3 \mathrm{~g}$ show that, compared with the control group, the SOD activity in the cells in the UVB group clearly decreased and the SOD activity in the cells incubated with added BVD before UVB irradiation was clearly increased, indicating a dosedependent correlation with BVD concentration; the difference for each group was statistically significant $(p<0.05)$. The result in Fig. 3h shows that, compared with the control group, the MDA content in the cells in the UVB group increased, with a statistically significant difference $(p<0.05)$, and that the MDA content of HaCaT cells in each group irradiated by UVB after BVD pretreatment was reduced. However, only the difference between the $10 \mu \mathrm{mol} / L$ BVD + UVB group and the UVB group was statistically significant $(p<$ 0.05).

Effect of BVD on MMP-1 and MMP-3 protein expression in UVB-irradiated HaCaT cells

The western blot results showed that, compared with the control group, MMP-1(Fig. 4a) and MMP3(Fig. 4b) expression in the UVB group increased, MMP-1 and MMP-3 expression in the HaCaT cells in each group with BVD pretreatment and UVB irradiation decreased $(p<0.05)$, and the differences in MMP1 and MMP-3 expression for the $0.1 \mu \mathrm{mol} / \mathrm{L}, 1 \mu \mathrm{mol} / \mathrm{L}$, and $10 \mu \mathrm{mol} / \mathrm{L} B V D+U V B$ groups were all statistically significant $(p<0.05)$.

Effect of BVD on Nrf2 protein expression in UVB-irradiated HaCaT cells

The western blot results showed in Fig. 5, compared with the control group, Nrf2 protein expression in the HaCaT cells of the UVB group decreased $(p<0.05)$ but Nrf2 protein expression in the $0.1 \mu \mathrm{mol} / \mathrm{L}$, $1 \mu \mathrm{mol} / \mathrm{L}$, and $10 \mu \mathrm{mol} / \mathrm{L}$ BVD + UVB groups increased; the protein expression in each BVD + UVB group increased compared with that in the UVB group $(p<0.01)$.

Effect of Nrf2 gene enhancement on Nrf2 protein expression and viability in UVB-irradiated HaCaT cells 
The western blot results showed in Fig. 6a, Nrf2 protein expression was reduced in HaCaT cells after UVB irradiation, and Nrf2 protein expression in Nrf2-shRNA stably transfected HaCaT cells was increased. The CCK-8 results showed that the cell viability of experimental groups was $100 \%, 21.06 \%, 71.25 \%$, and $43.04 \%$ (Fig. 6b), respectively. Compared with the control group (no transfection, no UVB irradiation), cell activity in the UVB group was clearly reduced; the viability of cells in the shRNA + UVB group was clearly increased compared with that of cells in the UVB group, and the difference was statistically significant ( $p$ $<0.05)$. These results illustrate that Nrf2 gene enhancement increased the viability in UVB-irradiated HaCaT cells.

\section{Discussion}

Oxidative stress plays a key role in the pathogenesis of photoaging,and some studies have confirmed that antioxidants are very effective to prevent skin photoaging in recent years [23, 24]. In this experient, the defense mechanism of BVD on the photodamage caused by UVB irradiation was explored in HaCaT cell. Both BVD and the metabolite BIL have antioxidation effects [25]. Under these in vitro conditions, BVD scavenges peroxyl radicals more effectively than unconjugatedor conjugated BIL: each molecule of BIL and BVD scavenges 1.9 and 4.7 molecules of peroxyl radicals, respectively. In addition $\square B V D / B I L$ have been reported to scavenge a number of other ROS, suggesting that BIL and BVD can scavenge both 1eand 2e-oxidants, similar toascorbate [26]. By comparison, many other antioxidants are effective against only one class of oxidants. For example, atocopherol reacts rapidly with 1e-oxidants, but is a poor scavenger of 2e-oxidants [27]. In this study, we found that BVD had a protective effect against UVBinduced photodamage. Compared with cells in the control group, after $30 \mathrm{~mJ} / \mathrm{cm}^{2}$ UVB irradiation and a 24-hour culture, HaCaT cells became smaller and rounded, and the number of cells was clearly reduced. Moreover, as the BVD concentration increased, the cells pretreated with BVD had less cell damage, showing a dose correlation, which fully proved that BVD is able to increase the viability of UVB-irradiated cells.

Overexposure to UVB irradiation results in the overproduction of free radicals, playing a potentially important role in damaged skin, such as photo-damage, photo-aging and skin cancer. Free radicals can diminish the protection of antioxidant enzymatic activities, resulting in increased oxidative stress. During oxidative stress, ROS and MDA are continuously being produced, and SOD is being decreased [28-30]. In this study, it was shown that the ROS in cells clearly increased after UV irradiation but that ROS decreased significantly in cells incubated for one hour with BVD before UV irradiation, indicating a dosedependent correlation with BVD concentration. In this study, it was also shown that intracellular SOD activity clearly reduced after UV irradiation; after adding BVD at a certain concentration, SOD activity increased. In addition, this study showed that UVB irradiation increased the MDA levels in HaCaT cells and that the MDA levels in the UVB group pretreated with BVD were clearly reduced. The above results show that the ROS and MDA levels in cells in the BVD pretreatment groups were clearly reduced and the SOD activity was significantly increased, thereby proving that BVD plays an antioxidative role in UVBinduced photodamaged cells. 
MMPs are secreted by keratinocytes and dermal fibroblasts after UV irradiation. wherein the abnormal expression of MMPs plays a major role in the pathophysiological mechanism of skin photoaging [31, 32]. Long-term exposure to UV can reconstruct the extracellular matrix (ECM), degrade collagen, damage connective tissue, and generate a series of ROS, leading to premature photoaging of the skin [33]. The changes start from the activation of MMPs, ultimately leading to the generation of skin wrinkles [34]. Therefore, inhibiting MMPs generation has become an important strategy in the prevention of photodamage, and reducing the natural materials produced by MMPs can similarly prevent photodamage $[35,36]$. In this study, it was found that after keratinocytes were UV irradiated, MMP-1 and MMP-3 protein expression increased, whereas MMP-1 and MMP-3 protein expression in cells in the UVB group pretreated with BVD was clearly inhibited, showing a dose correlation, which further confirms that BVD has a protective effect against UVB-induced cell photodamage.

Nrf2 participates in the regulation of the cellular antioxidant response through the Nrf2/ARE pathway [37]. When the external redox environment changes or is induced by the signal transduction system, this pathway can be activated to switch on downstream target genes that play roles in antioxidation, antiinflammation, antiapoptosis, maintaining the stability of the internal environment, and so on. The Nrf2/ARE pathway plays a role in numerous diseases associated with the oxidative stress response [3840]. In this study, we found that after enhancing the Nrf2 gene in keratinocytes is via lentivirus transfection, Nrf2 protein expression was significantly increased, and Nrf2 protein expression and viability of cells in the shRNA + UVB group were clearly higher than those of the cells in the UVB group, which further illustrates that the application of Nrf2 activators can increase the level of intracellular Nrf2 protein expression and the viability of UVB-irradiated cells. In a study by Soeur $\mathrm{J}$ et al., after UV irradiation was performed on Nrf2 gene knockout mice and antioxidative gene expression was reduced, the oxidative stress damage was clearly enhanced, thereby proving that the Nrf2-ARE pathway is important for regulating the intracellular redox status[13], which is consistent with our study. In this study, after the keratinocytes were UVB irradiated, Nrf2 protein expression was reduced, and cell viability was clearly reduced; however, Nrf2 protein expression was clearly increased and cell viability was clearly increased after UVB-irradiated cells were pretreated with BVD. This illustrates that BVD can activate the Nrf2 protein to antagonize the cell photodamage caused by UVB irradiation and that its mechanism may be related to the Nrf2 antioxidative stress pathway.

\section{Conclusion}

In summary, UVB irradiation can lead to a clear decrease in the viability of HaCaT cells, but the viability of cells in the UVB group pretreated with BVD was significantly increased, suggesting that BVD can antagonize the cellular photodamage caused by UVB irradiation. The Nrf2 gene has a protective effect against photodamage. As an activator of Nrf2, BVD has a protective effect in UVB-irradiated HaCaT cells, and its mechanism is related to the Nrf2 antioxidative stress pathway.

\section{Methods}


Chemicals

BVD HCl (Sigma Company, USA.10 mg; No. 30891) was dissolved in $0.2 \mathrm{~N} \mathrm{NaOH}$, adjusted to a final pH of 7.4 with $\mathrm{HCl}$ and subsequently diluted with saline $(0.9 \% \mathrm{NaCl})$ to the final concentrations. The final $\mathrm{pH}$ was between 7.3 and 7.5 for each BVD solution used in this study.

Cell Culture

$\mathrm{HaCaT}$ cells were provided by the laboratory of the Second Affiliated Hospital of Harbin Medical University. The immortalized human keratinocytes, $\mathrm{HaCaT}$, were cultured in DMEM containing $4.5 \mathrm{~g} \mathrm{~L}^{-1}$ glucose and L-glutamine supplemented with $10 \%$ fetal bovine serum, $100 \mu \mathrm{g} \mathrm{mL}^{-1}$ penicillinand $100 \mathrm{U}$ $\mathrm{mL}^{-1}$ streptomycin. The cells were maintained under standard cell culture conditions at $37^{\circ} \mathrm{C}$ and $5 \%$ $\mathrm{CO} 2$ in a humid environment.

Cell Grouping And Prossing

HaCaT cells were divided into five groups: control group: no BVD added, no UVB irradiation; UVB group: $30 \mathrm{~mJ} / \mathrm{cm}^{2}$ UVB irradiation, no BVD added; and three BVD groups $-100 \mathrm{nmol} / \mathrm{L}, 1 \mu \mathrm{mol} / \mathrm{L}$, or $10 \mu \mathrm{mol} / \mathrm{L}$ $B V D+U V B$ groups: corresponding concentration of BVD was added one hour before $30 \mathrm{~mJ} / \mathrm{cm}^{2} \mathrm{UVB}$ irradiation.

HaCaT cells were divided into four groups: control group: no transfection, no UVB irradiation; UVB group: $30 \mathrm{~mJ} / \mathrm{cm}^{2}$ UVB irradiation, no transfection; shRNA group:lentivirus transfection and gene enhancement, no UVB irradiation; and shRNA + UVB group: lentivirus transfection and gene enhancement before $30 \mathrm{~mJ} / \mathrm{cm}^{2}$ UVB irradiation.

UVB Irradiation

A bank of two UVB lamps (Nanjing huaqiang, China) was used as the UVB source. These lamps emit UV light at wavelengths ranging from 290 to $320 \mathrm{~nm}$ (mainly UVB), with the peak emission at $313 \mathrm{~nm}$. The intensity of irradiation by the lamp $\left(3.0 \mathrm{~mW} / \mathrm{m}^{2}\right)$ was measured using a Waldmann UV meter (Waldmann, Medizintechnik, Germany). The cells to be irradiated by UVB were prepared. When their confluence reached more than $80 \%$, the culture solution was aspirated, PBS was used to wash the cells once, and a small amount of PBS was added to cover the bottom surface of the plate; UVB irradiation (total irradiation dose of $30 \mathrm{~mJ} / \mathrm{cm}^{2}$ ) was applied with the culture plate $15 \mathrm{~cm}$ from the light source. After irradiation, the PBS was discarded, and fresh DMEM was added, and the cells were cultured for 24 hours, after which the cells were collected for analysis.

Virus Transfection

The infection method and infection parameters were confirmed by an interference pretest. Fluorescence was used to determine the multiplicity of infection (MOI), 20. During infection, the addition of polybrene 
was the most suitable method. At 48 hours after infection, irradiation was carried out on the cells according to the cell growth situation.

CCK-8 Method For The Detection Of Cell Viability

After culturing cells for 24 hours, $10 \mu \mathrm{l}$ of CCK8 (Tongren Company, Japan)was added into each well, and cell culture was continued for 4 hours. The absorbance (A value) of each well was measured by an enzyme-labeling instrument at a wavelength of $450 \mathrm{~nm}$. Cell viability=(experimental well A value-blank well A value)/(control well A value-blank well A value) $\times 100 \%$.

Detection Of Cellular ROS, SOD and MDA

Detection of cellular ROS carried out in accordance with the kit instructions (Biyuntian Company, Shanghai ,China). Detection of cellular SOD and MDA carried out in accordance with the kit instructions (Jiancheng Bio-Technology, Nanjing, China).

Western blot detection of MMP-1, MMP-3, and Nrf2 protein expression

The MMP-1, MMP-3 and Nrf-2 protein levels were detected by Western blot. Protein samples were extracted. Protein extract was boiled in loading buffer for $5 \mathrm{~min}$, separated using SDS-polyacrylamide gel electrophoresis and then transferred to polyvinylidene fluoride (PVDF) membranes. The membranes were blocked in $5 \%$ nonfat dried milk overnight and then incubated with primary rabbit polyclonal Nrf2, antiMMP-1 anti-MMP-3 antibody (1:500, Santa Cruz Biotechnology, Santa Cruz, CA) for $2 \mathrm{~h}$ followed by secondary horseradish peroxidase-tagged goat anti-rabbit antibody (1:500, Zhongshan Golden Bridge Biotechnology, Beijing, China) for $1 \mathrm{~h}$. Finally, the membranes were developed with 3, 30diaminobenzidine reagent and exposed to film. Protein levels were normalized to the corresponding bactin protein levels (1:500; Zhongshan Golden Bridge Biotechnology, Beijing, China). The relative expression levels of each protein were expressed as the ratio of signal intensity of the target proteins to that of the ß-actin.

Statistical analysis

Statistical analysis of data was performed by experts, using SPSS 17.0 software. The data are expressed as the mean \pm standard deviation (SD). Differences in measured variables between groups were determined by one-way analysis of variance (ANOVA) followed bya Student-Newman-Keuls (SNK) test. A significant difference was set at $p<0.05$.

\section{Abbreviations}

BIL:Bilirubin;BVD:Biliverdin;HO-1:heme oxygenase-1;MDA:malondialdehyde;MMPs:matrix metalloproteinases;Nrf2:nuclear factor erythroid 2-related factor 2;ROS:reactive oxygen and species;SOD:superoxide dismutase 


\section{Declarations}

Acknowledgements

The authors thank the support of the experiment of the second affiliated hospital of Harbin medical university.

\section{Authors' contributions}

Ma Liangjuan,Gong Jie,Liu Yingdi,Ren Jing,Lin Fang,Zhang Xiaohui,You Yan,Bai Bingxue and Li Yuzhen performed the experiments and analyzed data together.Gong Jie wrote the manuscript, with contributions from Ma Liangjuan. All authors read and approved the final manuscript.

\section{Funding}

This work is supported by the national natural science foundation of China囚81502706ه.

\section{Availability of data and materials}

The datasets used and/or analyzed during the current study are available from the corresponding author on reasonable request

\section{Ethics approval and consent to participate}

Not applicable.

\section{Consent for publication}

Not applicable.

\section{Competing interests}

The authors declare that they have no competing interests.

\section{Author details}

${ }^{1}$ The Department of Dermatology, The Second affiliated Hospital of Harbin Medical University, Harbin 150081, Heilongjiang Province, People's Republic of China. ${ }^{2}$ The Department of Dermatology, Chifeng Municipal Hospital, Hongshan district, Chifeng 024000, Neimenggu Province,People's Republic of China. ${ }^{3}$ The Department of Dermatology,Caoxian People's Hospital,Caoxian development zone 274400, Shandong province $『$ People's Republic of China. ${ }^{4}$ The Department of Dermatology,CangzhouCentral Hospital, Cangzhou 061000, Hebei province,People's Republic of China. ${ }^{5}$ The Department of Dermatology,WeifangTraditional Chinese Hospital, Weifang 261041, Shandong province, People's Republic of China. ${ }^{6}$ The Department of Dermatology, The Fourth affiliated Hospital of Harbin Medical University, Harbin 150040, Heilongjiang Province, People's Republic of China . 


\section{References}

1.Tiejun Chen, Hu Hou, Jiaohan Lu, Kai Zhang,Bafang Li.Protective effect of gelatin and gelatin hydrolysate from salmon skin on UV irradiation-induced photoaging of mice skin. Journal of Ocean University of China.2016;15:711-718.

2.Pérez-Sánchez,A.Barrajón-Catalán,E.Herranz-López,M.Micol.Nutraceuticals for skin care: A comprehensive review of human clinical studies.Nutrients.2018; 24:10(4).

3.PoonF,KangS,Chien AL.Mechanisms and treatments of photoaging.Photodermatol Photoimmunol Photomed.2015;31:65-74.

4.Gilgun-Sherki Y, Rosenbaum Z, MelamedE,Offen D.Antioxidant therapy in acute central nervous system injury:currentstate.Pharmacol Rev .2002;54:271-284.

5.Akinyemi, A. J., Ademiluyi, A. O. ,Oboh, G.Aqueous extracts of two varieties of ginger (Zingiberofficinale) inhibit angiotensin I-converting enzyme, iron(II), and sodium nitroprusside-induced lipid peroxidation in the rat heart in vitro.J Med Food.2013;16:641-6.

6.Olalekan Lawal A, Lawal AF, Ologundudu A, Adeniran OY, Omonkhua A,Obi F.Antioxidant effects of heated garlic juice on cadmium induceliver damage in rats as compared to ascorbic acid. J Toxicol Sci.2011;36:549-57.

7.Hao yongjian, liubing ,qinxiankui.Effect of tunnel airtight working environment on antioxidant enzyme and lipid peroxide activity of officers and soldiers serum.PLA journal of preventive medicine.2018; 36:1350-1351.

8.Galliera E,TacchiniL,CorsiRomanelli MM.Matrix metalloproteinasesas biomarkers of disease:updates and new insights. 2015;53:349-55.

9.Kammeyer A, Luiten R M.Oxidation events and skin aging. Ageing Res Rev 21:16-29

10.Schafer M., Werner S.Nrf2--A regulator of keratinocyte redox signaling. Free Radic Biol Med. 2015;88,243-252.

11.Xu N,Huang F,Jian C,Qin L,Lu F,Wang Y,Zhang Z,Zhang Q.Neuroprotective effect of salidroside against central nervous system inflammation-induced cognitive deficits: A pivotal role of sirtuin 1dependent Nrf-2/HO-1/NF-KB pathway.Phytother Res .2019;33:1438-1447.

12.Gęgotek A , Skrzydlewska E.The role of transcription factor Nrf2 in skin cells metabolism.Arch Dermatol Res.2015; 307(5):385-96.

13.Soeur J, Eilstein J, Léreaux G, Jones C,Marrot L.Skin resistance to oxidative stress induced by resveratrol: from Nrf-2 activation to GSH biosynthesis. Free Radic Biol Med .2015;78:213-23. 
14.Reisman SA, Lee CY, Meyer CJ, Proksch JW, Ward KW.Topical application of the synthetic triterpenoid RTA 408 activates Nrf2 and induces cytoprotective genes in rat skin.Arch Dermatol Res.2014;306(5):44754.

15.Kim M , Park YG , Lee HJ , Lim SJ,Nho CW .Youngiasides A and C isolated from youngiadenticulatum inhibit UVB囚induced MMP expression and promote type I Procollagen production via repression of MAPK/AP®1/NF『kB and activation of AMPK/Nrf2 in HaCaT cells and human dermal fibroblasts.JAgric Food Chem .2015;63:5428ه5438.

16.Tian WF, Weng P, Sheng Q, Chen JL, Zhang P, Zhang JR, Du B, Wu MC, Pang QF ,Chu JJ.Biliverdin Protects the Isolated Rat Lungs from Ischemia-reperfusion Injury via Antioxidative, Antiinflammatory and Anti-apoptotic Effects.Chin Med J (Engl).Chin Med J (Engl).2017;130:859-865.

17.Spetzler VN, Goldaracena N, Kaths JM, Marquez M, Selzner N, Cattral MS, Greig PD, Lilly L, McGilvray ID, Levy GA, Ghanekar A, Renner EL, Grant DR,Selzner M.High preoperative bilirubin values protect against reperfusion injury after live donor liver transplantation.Transpl Int.2015;28:1317-25.

18.Hettiarachchi N, Dallas M, Al-Owais M, Griffiths H, Hooper N, Scragg J, Boyle J, Peers C.Heme oxygenase-1 protects against Alzheimer's amyloid- $\beta(1-42)$-induced toxicity via carbon monoxide production.Cell Death Dis.2104;5:e1569.

19.Lerner-MarmaROSh N, Shen J, Torno M D, Kravets A, Hu Z,Maines MD.Human biliverdinreductase: a member of the insulin receptor substrate family with serine/threonine/tyROSine kinase activity. Proc Natl Acad Sci U S A .2005;102:7109-14.

20.Masnec I S, Poduje S.Photoaging. Coll Antropol.2008; 2:177-80.

21.Junko Kosaka, Hiroshi Morimatsu, Toru Takahashi, Hiroko Shimizu, Susumu Kawanishi, Emiko Omori, Yasumasa Endo, Naofumi Tamaki, Manabu Morita, Kiyoshi Morita.Effects of Biliverdin Administration on Acute Lung Injury Induced by Hemorrhagic Shock and Resuscitation in Rats. LoS One.2013;7:8(5).

22.Bai B, Liu Y, You Y, Li Y,Ma L.Intraperitoneally administered biliverdin protects against UVB-induced skin photo-damage in hairless mice.J Photochem Photobiol B.2015;144:35-41.

23.Burke KE.Photoaging: the role of oxidative stress.G Ital Dermatol Venereol.2010;145:445-59.

24.Bosch R, Philips N, Suárez-Pérez JA, Juarranz A, Devmurari A, Chalensouk-Khaosaat J,González S.Mechanisms of Photoaging and Cutaneous Photocarcinogenesis, and Photoprotective Strategies with Phytochemicals. Antioxidants (Basel).2015;4:248-68.

25.MacLean PD , Chapman EE, Dobrowolski SL, Thompson A,Barclay LR.Pyrroles as antioxidants: solvent effects and the nature of the attacking radical on antioxidant activities and mechanisms of pyrroles,dipyrrinones, and bile pigments. J Org Chem .2008;73:6623-6635. 
26.Carr AC, Zhu BZ, and Frei B.Potential antiatherogenic mechanisms of ascorbate (vitamin C) and alphatocopherol (vitamin E). Circ Res.2000; 87:349-54.

27.Stocker R.Antioxidant activities of bile pigments.AntioxidRedox Signal.2004; 6:841-9

28.Hongkun Wang , Yanhui Zhang , Fangfang Xia, Wei Zhang, Peng Chenand Guoan Yang.Protective effect of silencing Stat1 on high glucose-induced podocytes injury via Forkhead transcription factor 01regulated the oxidative stress response.BMC Molecular and Cell Biology. (2019) 20:27.

29.Qiang Wan, Zhongyong Liu, Ming Yang , Peng Deng , Nana Tang and Yanwei Liu.Triptolide ameliorates fine particulate matter-induced podocytes injury via regulating NF-KB signaling pathway.BMC Molecular and Cell Biology. (2020) 21:4.

30.Yang $\mathrm{w} \mathrm{J}$, sun $\mathrm{y}$,yuan b.Immune regulation and antioxidant function of ultrafine powder of edible fungi . Acta bacteriologica sinica.2015; 34:301-308.

31.Im AR, Ji KY, Park I, Lee JY, Kim KM, Na M,Chae S. Anti-Photoaging Effects of Four Insect Extracts by Downregulating Matrix Metalloproteinase Expression via Mitogen-Activated Protein Kinase-Dependent Signaling.Nutrients.2019;23;11(5).

32.Kerkelä E, Saarialho-KereU.Matrix metalloproteinases in tumor progression: focus on basal and squamous cell skin cancer. ExpDermatol.2010; 12:109-25.

33.P. Pittayapruek, J. Meephansan, O. Prapapan, M. Komine and M.Ohtsuki.Role of Matrix Metalloproteinases in Photoaging and Photocarcinogenesis.Int J Mol Sci.2016; 17:868.

34.Quan T, Qin Z, Xia W, Shao Y, Voorhees JJ,Fisher GJ.Matrix-degrading metalloproteinases in photoaging.J Investig Dermatol Symp Proc .2009;14:20-4.

35.López-López N , González-Curiel I, Treviño-Santa Cruz MB, Rivas-Santiago B, Trujillo-Paez V, EncisoMoreno JA,Serrano CJ.Expression and vitamin D-mediated regulation of matrix metalloproteinases (MMPs) and tissue inhibitors of metalloproteinases (TIMPs) in healthy skin and in diabetic foot ulcers.Arch Dermatol Res.2014; 306(9):809-21.

36.Lu Li, Eunson Hwang, Hien T. T. Ngo, Seul A. Seo, Pei Lin, Wei Gao, Ying Liu and Tae-Hoo Yi(2018)Ribesnigrum L. Prevents UVB-mediated Photoaging in Human Dermal Fibroblasts: Potential Antioxidant and Anti-inflammatory Activity.PhotochemPhotobiol 94:1032-1039.

37.Guo H , Zheng Y , Wang BLi Z. A note on an improved self \healing group key distribution scheme.Sensors (Basel) .2015;15:25033-8.

38.Muthusamy VR, Kannan S, Sadhaasivam K, Gounder SS, Davidson CJ, Boeheme C, Hoidal JR, Wang L,Rajasekaran NS.Acute exercise stress activates Nrf2/ARE signaling and promotes antioxidant mechanisms in the myocardium. Free Radic Biol Med .2012;52:366-76. 
39.Jiménez-Vidal L,Espitia-Pérez P,Torres-Ávila J,Ricardo-Caldera D, Salcedo-Arteaga S, Galeano-Páez C ,Pastor-Sierra K, Espitia-Pérez L .Nuclear factor erythroid 2 - related factor 2 and its relationship with cellular response in nickel exposure: a systems biology analysis.BMC Pharmacol Toxicol. 2019 Dec 19;20(Suppl 1):78.

40.Heo HS, Han GE, Won J, Cho Y, Woo H,Lee JH.Pueraria montana var. lobata root extract inhibits photoaging on skin through Nrf2 pathway. J Microbiol Biotechnol .2019;29:518-526.

\section{Figures}

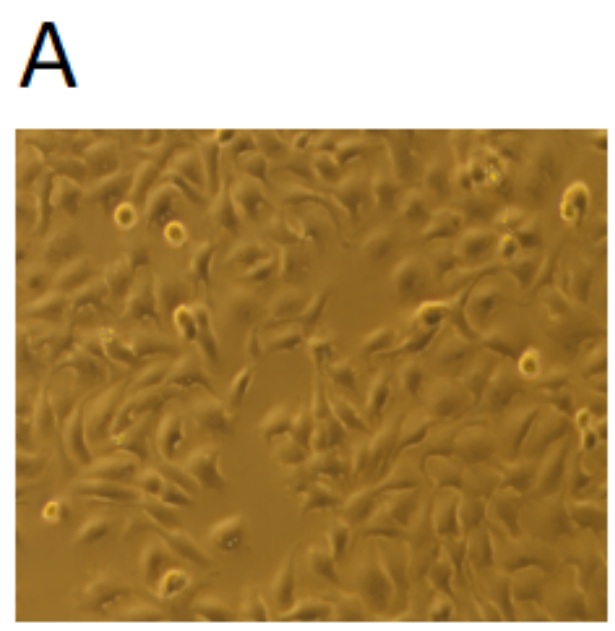

B

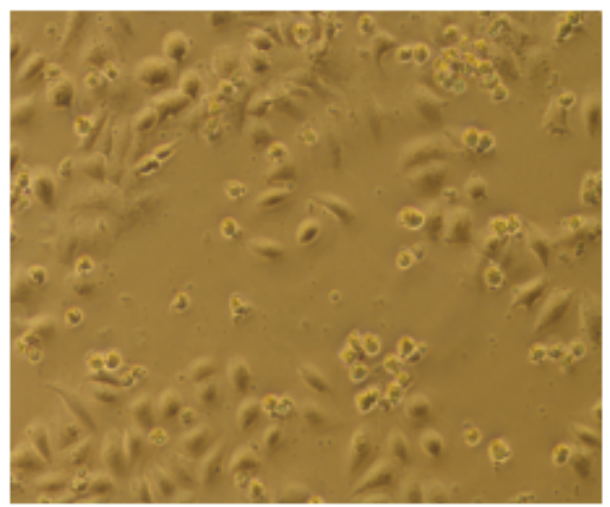

\section{Figure 1}

Cell morphology under an optical microscope (×200)『HaCaT cells with normal morphology (a); Morphology of HaCaT cells cultured for 24 hours after irradiation with $30 \mathrm{~mJ} / \mathrm{cm} 2 \mathrm{UVB}(\mathrm{b})$. 


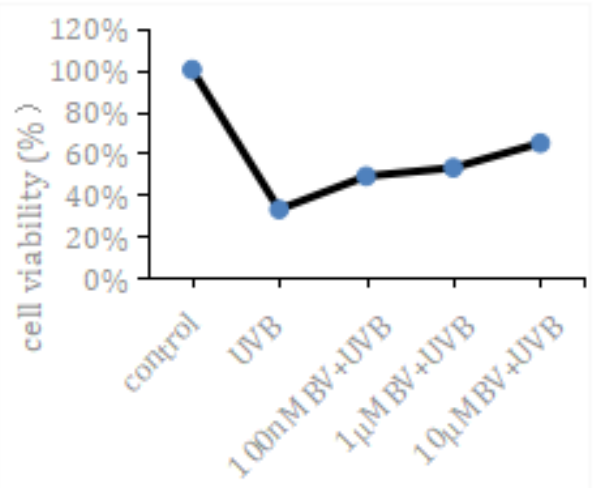

Figure 2

Cell viability of each group $\triangle$ Compared with the control group, cell activity in the UVB group was clearly reduced $\llbracket p<0.05 \rrbracket$; cell activity in the BVD+UVB groups was clearly increased, vs the UVB group $\llbracket p<0.05 \rrbracket$; cell activity in three BVD+UVB groups was statistically significant $(p<0.05)$.
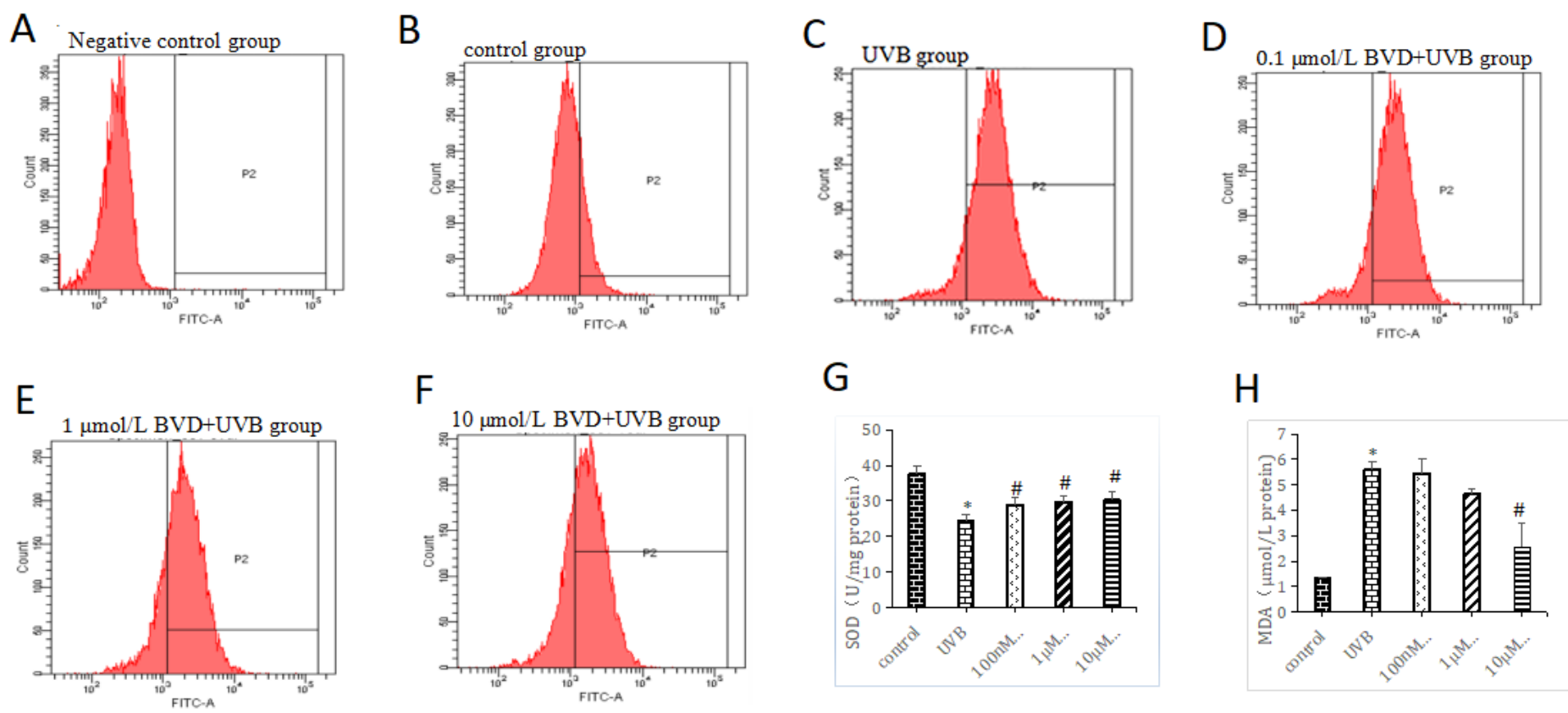

Figure 3

Cellular ROS fluorescence intensity (MEAN) in experimental groups was1645, 3403, 2904, 2689, 2382(bf). Compared with the control group, the MEAN ROS fluorescence intensity of the cells in the UVB group clearly increased, ROS level (MEAN) of the cells incubated with BVD prior to UVB irradiation was clearly lower than that of the UVB group. Intracellular SOD activity in each group $\varangle * p<0.05$, vs control group; $\# p<0.05$, vs UVB group, $n=3(\mathrm{~g})$. Intracellular MDA content in each group $\triangle * \mathrm{p}<0.05$, vs control group; $\# p<0.05 \bigotimes$ only the $10 \mu \mathrm{mol} / L$ BVD+UVB group $\rrbracket$, vs UVB group, $\mathrm{n}=3$ (h). 
A

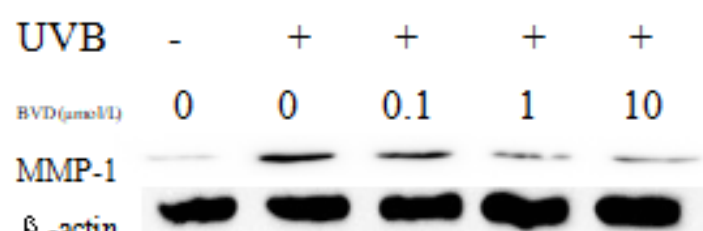

B

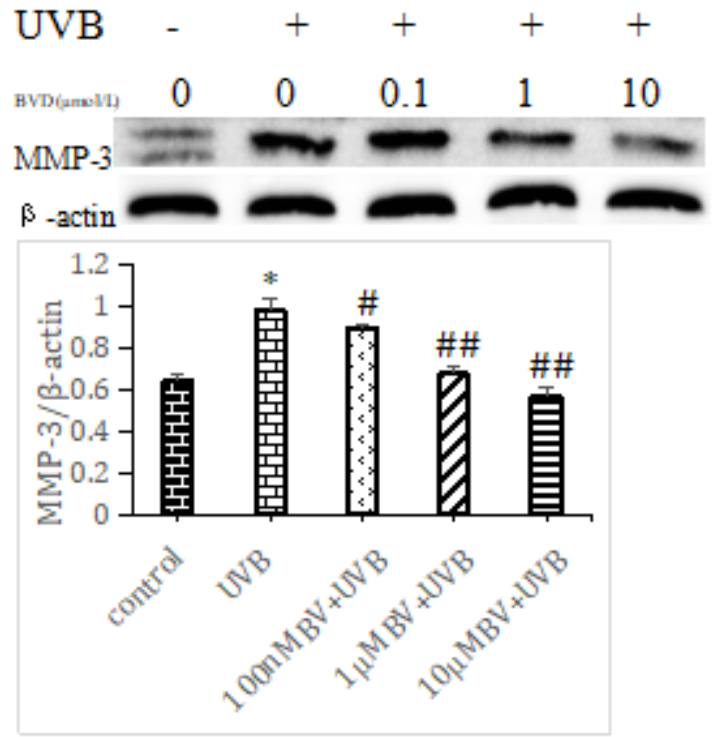

Figure 4

Intracellular MMP-1 protein expression in each group:* $p<0.01$, vs control group; $\# p<0.01$, vs UVB group, $n=3(a)$. Intracellular MMP-3 protein expression in each group: ${ }^{*}<<0.01$, vs control group; $\# p<0.05$, vs UVB group; \#\#p<0.01, vs UVB group, $n=3(b)$.

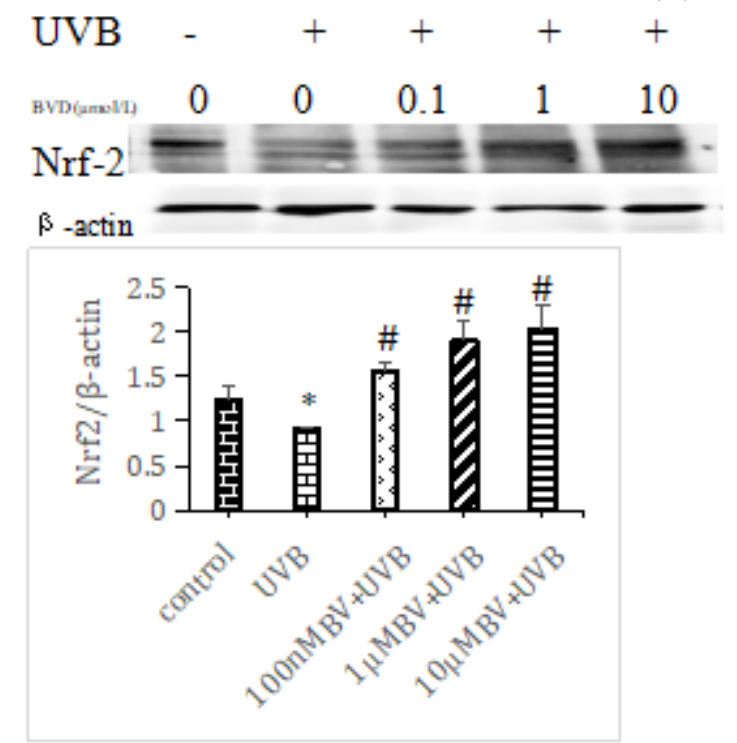

Figure 5

Nrf2 protein expression in the cells in each group: ${ }^{*}<<0.05$, vs control group; $\# p<0.01$, vs UVB group, $n=3$. 


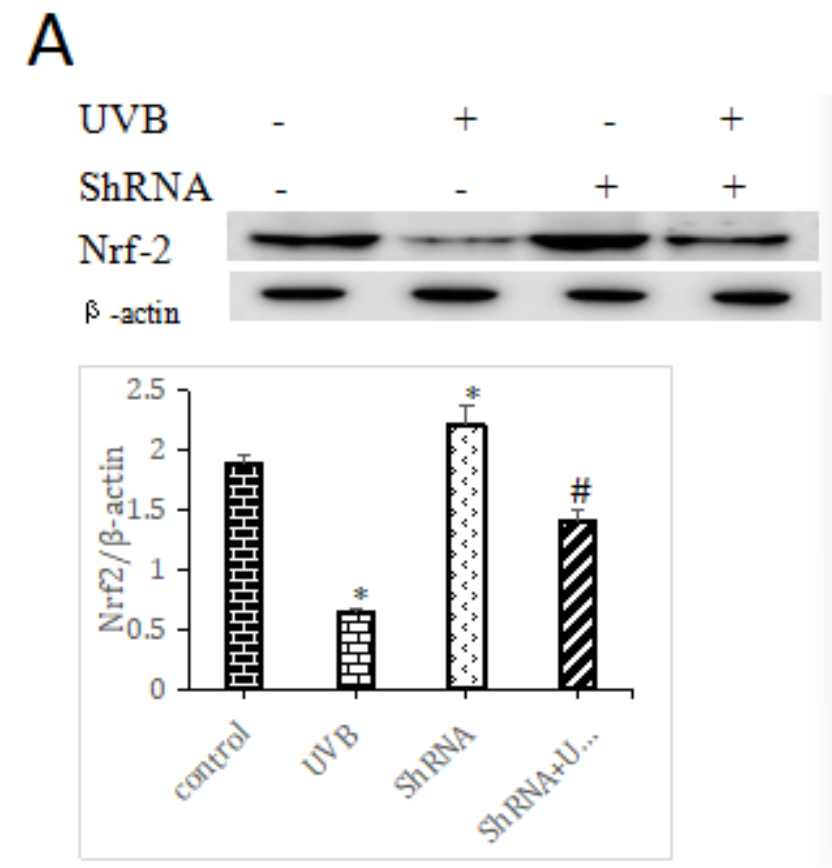

B

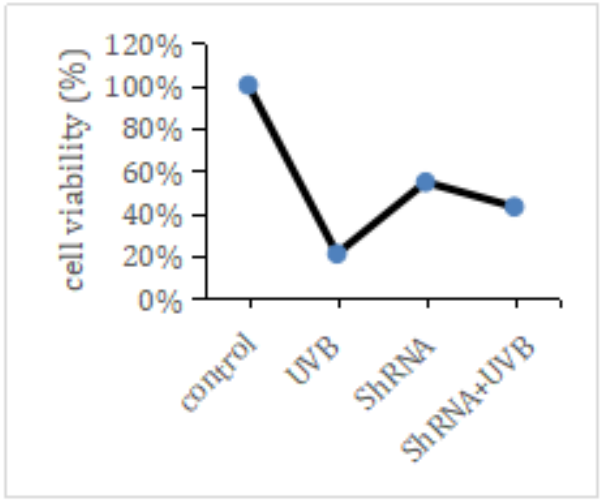

\section{Figure 6}

Nrf2 protein expression in each group $\nabla^{\star} p<0.05$ vs control group; $\# p<0.05$ vs UVB group, $n=3(a)$. Cell viability in each group $₫$ for UVB group $\llbracket p<0.05$, vs control group; for shRNA+UVB group $\llbracket p<0.05$, vs UVB group, $\mathrm{n}=12$ (b). 